\section{Toluidinblau-Färbung im Blut}

H. Baum

Institut für Laboratoriumsmedizin, Mikrobiologie und Blutdepot, Regionale Kliniken Holding RKH GmbH, Ludwigsburg, Deutschland

Englischer Begriff toluidine blue stain

Definition Färbung zur selektiven Darstellung der Granula in basophilen Granulozyten und Mastzellen.

Physikalisch-chemisches Prinzip Das luftgetrocknete Ausstrichpräparat wird 5 Minuten in einer $1 \%$ igen ToluidinblauLösung gefärbt und anschließend mit Wasser gründlich gespült. Dabei bindet $>$ Toluidinblau selektiv an die Granula basophiler Granulozyten und Mastzellen.

Einsatzgebiet Differenzierung basophiler Granulozyten und Mastzellen in Fällen, in denen die Standardfärbung keine eindeutigen Ergebnisse erzielt. So können bei Patienten mit myeloproliferativen Erkrankungen dysplastische Basophile auftreten, die in der $>$ Pappenheim-Färbung nicht oder nur schlecht differenzierbar, jedoch in der Toluidinblau-Färbung eindeutig als basophile Granulozyten identifizierbar sind.

Untersuchungsmaterial Ausstrichpräparat des Knochenmarks oder peripheren Blutes.

Instrumentierung Handmethode.

Praktikabilität - Automatisierung - Kosten Einfach durchzuführende Handmethode.

Bewertung - Methodenhierarchie (allg.) Die Methode wird nur verwendet, wenn in den Standardfärbemethoden keine Differenzierung der dysplastischen Basophilen oder Mastzellen möglich ist.

\section{Literatur}

Swirsky D, Bain BJ (2001) Erythrocyte and leukocyte cytochemistry - leukaemia classification. In: Lewis SM, Bain BJ, Bates I (Hrsg) Dacie and Lewis practical haematology, 9. Aufl. Churchill Livingstone, London, S 288-289 